\section{A FRAMEWORK FOR YOUR FUTURE}

The Perfect Dental Practice has been designed by business leaders and practice managers for practices that need a helping hand. YoYo Dental continues with the theme of offering straightforward practical advice to dental practices.

Ken Turley, YoYo Dental's founding Director, has listened to the dental industry over the past few years whilst he has been offering advice and support in infection control, where his ongoing no-nonsense approach to achieving compliancy to HTM 01-05 has been well received.

As an entrepreneurial business leader he has seen practices needing to earn more revenues and make greater profit, dentists wanting to improve their life/work balance and practice managers looking to improve policies and procedures to ensure compliancy to $\mathrm{CQC}$ requirements.

The Perfect Dental Practice provides a comprehensive and bespoke business to business service that provides an on-site business health check by business leaders to ensure a profitable business combined with ongoing on-site support from experienced practice managers to mentor and train the team to ensure a fully CQC compliant practice.

In this financial climate can you afford not to take advice, or the offer of a helping hand that will provide a simple, controlled framework for your dreams and your future?

Contact Ken Turley at YoYo Dental on 07799478287 and mention the $B D J$.

\section{STRONG DENTINE REPLACEMENT}

Riva Self Cure HV from Southern

Dental Industries (SDI) is a high viscosity, extremely strong self curing glass ionomer restorative, tough enough to resist surface indentation and strong enough to withstand substantial mechanical loads.

Riva Self Cure HV's packability

\title{
SMART ONLINE APPOINTMENT BOOKING
}

Software of Excellence (SoE) has produced an online appointment booking system that integrates with a dental practice's recall management.

This new approach has been developed as a direct response to feedback from dental professionals and features software that identifies patients due for recall on a daily basis according to defined criteria, preset by the practice. The software automatically sends recall messages via email or text with a link prompting the patient to access a practice-branded online booking facility, detailing available appointment times with the patient's own clinician. The patient selects the desired appointment and the software automatically identifies the patient and reserves the appointment, updating the practice's computerised appointment book in real time. Patients receive a confirmation email which can also be customised by the practice to include a map, an image of the practice and dentist,

\section{HIGH PRECISION SCALING}

The brand new high precision Powercare piezon scaler from Bien-Air is perfect for all prophylactic supragingival and sub-gingival scaling, and has proved to be extremely effective at removing all deposits.

The Powercare unit monitors and automatically adjusts the power needed for each operation. This guarantees optimal efficiency at all times and your patients benefit from increased comfort. opening hours, new treatments and can even be used to promote special offers. Experienced practices can also configure the software to 'smartly' select appointment times that they may traditionally find difficult to fill.

SoE's new online recall booking module provides a software solution that provides more than simply a mechanism for a patient who needs an appointment. This new technology is an integral part of the recall process that works via email and on smartphones, stimulating successful recall response rates and making a measurable and significant impact on the overall efficiency of a practice.

EXACT's Online Recall Booking Module is available from Software of Excellence, call 08003286227 or visit www.soeidental.com for more details.

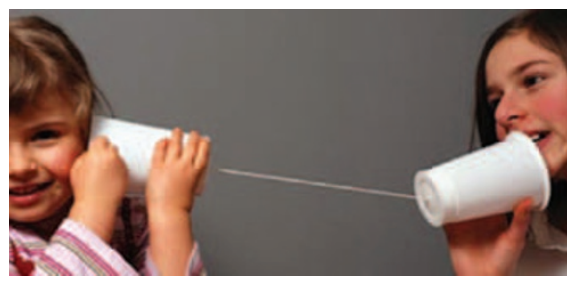

With its light ergonomically designed handle, Powercare offers you great visibility and ease of handling. With seven tips to choose from, this versatile piezon scaler can be purchased as a complete set. The Powercare set comprises: Powercare ultrasonic scaler, scaler hose, electronic control, steriliseable storage box, 2 x S1 scaling inserts, $\mathrm{P} 1$ universal periodontic insert and potentiometer.

For further information contact Bien-Air on 01293550200 or visit www.bienair.com. 